\title{
Does the duration of static stretching acutely interferes on the strength endurance performance?
}

\author{
Aline Alves da Silva, Emerson Luiz Teixeira, and Vitor de Salles Painelli* \\ Institute of Health Sciences, Paulista University, Sao Paulo, Brazil
}

Copyright: (C) 2019 A. A. da Silva et al. This is an open access article licensed under the Creative Commons Attribution License (https://creativecommons.org/licenses/by/4.0/).

Background: Stretching may promote detrimental effects on power output and maximal strength, but its direct effect on strength endurance remains unclear, as well as the influence of stretching duration. Objective: We aimed to evaluate the direct effect of static stretching of the quadriceps muscle, as well as the stretching duration, on the strength endurance during a unilateral knee extension exercise. Methods: Fifteen strength-trained men (age $28 \pm 3$ years, height $1.70 \pm 0.09 \mathrm{~m}$, body mass $70 \pm 5 \mathrm{~kg}$, training experience $5 \pm 1$ years) took part in a randomized counterbalanced cross-over study. Participants' total number of repetitions was assessed during strength endurance tests (four sets until failure at $70 \%$ of one-repetition maximum) performed under three different conditions in separate days: control - no stretching (CON), short-duration stretching (SDS) and long-duration stretching (LDS). Three stretching exercises, with 3 sets lasting 30 seconds each, were performed during SDS, while the number of sets during LDS was doubled. Data were analyzed using mixed models and magnitude-based inferences (MBI). Results: Despite of a significant decrease in the number of repetitions during the strength endurance test performed across the exercise sets (main Set effect $p<.001$ ), neither the number of repetitions in each set (main Condition effect $p=.95$ ) nor the total number of repetitions (main Condition effect $p=.86$ ) were significantly different between the conditions. MBI analysis revealed that changes in the total number of repetitions after SDS and LDS were mainly trivial compared to CON. Conclusions: Regardless of its duration, an acute session of static stretching did not influence strength endurance.

Keywords: muscle strength, exercise test, range of motion, stretching

\section{Introduction}

Flexibility is a sensory-motor capacity widely known to promote several benefits, both in the sport and health context (Garber et al., 2011). International recommendations have emerged in order to encourage the inclusion of stretching practices during exercise training routines. The current American College of Sports Medicine recommendation suggests that stretching should be included in an exercise program at least 2 to 3 times per week (Garber et al., 2011). Despite the lack of a specific number of exercises, it is also recommended that 2 to 4 sets of stretching should be performed, where a given position should be held for 10 to 30 seconds until the point of slight discomfort, accumulating 60 seconds for each exercise. Thus, the

\footnotetext{
* Address for correspondence: Vitor de Salles Painelli, Strength Training Study and Research Group, Institute of Health Sciences, Paulista University, R. Vergueiro 1211 - Paraíso, 01504-000, Sao Paulo, SP, Brazil. E-mail: vitor.painelli@usp.br
}

stretching session should have an average duration of 6 to 8 minutes in the case of 2 to 3 exercises are employed for a specific muscle group (Garber et al., 2011).

The moment which stretching should be inserted into an exercise session is still a matter of intense debate, especially when combined with a strength training program. Studies have suggested that there may be an acute interference effect on force production when strength exercises are immediately preceded by stretching (Marek et al., 2005). Such suggestions are supported by systematic reviews (Shrier, 2004), which demonstrated that stretching performed before the strength exercises resulted in an average reduction of 10 to $30 \%$ in strength performance, mainly assessed by dynamic or isokinetic tests (i.e., one-repetition maximum test; or maximum voluntary contraction, respectively). Despite the need for further clarification, potential mechanisms that may help to explain this acute interference effect include changes in the length-tension relationship of skeletal muscle as well as 
in the viscoelastic properties of the muscle (Marek et al., 2005; Shrier, 2004).

Although the current body of evidence indicates that there may be an acute interference of stretching on maximal force production capacity, it is still uncertain whether stretching influence the total volume of exercise performed within a session (i.e., strength endurance). Some studies showed a decreased total volume of exercise after stretching (Barroso, Tricoli, Santos Gil, Ugrinowitsch, \& Roschel, 2012; Gomes, Simão, Marques, Costa, \& da Silva Novaes, 2011), but some studies did not (Heisey \& Kingsley, 2016; Ribeiro et al., 2014). It is known that the total volume of exercise is one of the most crucial factors to optimize muscle strength and hypertrophy (Robbins, Marshall, \& McEwen, 2012; Sooneste, Tanimoto, Kakigi, Saga, \& Katamoto, 2013). Thus, any intervention that may acutely interfere on the total volume of exercise could interfere with muscle hypertrophy (Junior, Berton, de Souza, Chacon-Mikahil, \& Cavaglieri, 2017).

In this sense, determining the real influence of stretching on total exercise volume, essentially, has practical relevance, both in the clinical and sport context. If indeed this strategy could acutely interfere on total volume of exercise, it would be expected that the longer the duration of the stretching, the greater the magnitude of the interference; especially because it has been speculated that increased stretching durations may lead to an unidentified central nervous system inhibitory mechanism, as stretching-induced decreased muscle activation has already been identified by previous studies through surface electromyography and the twitch interpolation technique (Alizadeh Ebadi \& Çetin, 2018; Islamoglu, Atan, Unver, \& Cavusoglu, 2016; Rossi, Pereira, Simão, Brandalize, \& Gomes, 2010). In fact, according to a recent meta-analysis, this hypothesis seems to prevail for power-related tasks (Simic, Sarabon, \& Markovic, 2013). However, to our knowledge, no study has investigated the influence of stretching duration on strength endurance. Therefore, the aim of the present study was twofold: 1) to examine the acute effect of a static stretching session of the quadriceps muscle group on subsequent strength endurance during a unilateral knee extension exercise; 2) to investigate the influence of stretching duration of quadriceps muscle group on subsequent strength endurance during a unilateral knee extension exercise. We hypothesized that an acute session of static stretching would promote a deleterious effect on subsequent strength performance; and that the longer the stretching duration, the worse the performance would be.

\section{Methods}

\section{Participants}

Fifteen healthy, young and recreationally strengthtrained men (age $28 \pm 3$ years, height $1.70 \pm 0.09$ $\mathrm{m}$, body weight $70 \pm 5 \mathrm{~kg}$, training experience $5 \pm 1$ years, training frequency $5 \pm 1$ days/week, unilateral knee extension maximum dynamic strength $98 \pm 10.5$ $\mathrm{kg}$ ) took part in this study. To participate, individuals should be between 18 and 45 years old, with an uninterrupted previous experience in strength training of two years minimum, and without any orthopedic disturbance limiting the performance procedures. The exclusion criteria included the usage of any dietary supplements for at least two months prior to the study and any previous administration of anabolic steroids. Due to the exploratory nature of the current study, as well as to the absence of previous investigations examining the effects of the proposed intervention in this population, no sample power analysis was conducted a priori (Julious, 2005). All participants were fully informed of the risks and discomforts before giving their written informed consent. The study was approved by the institutional review board (approval number 80497717.1.0000.5512).

\section{Experimental design}

The protocol consisted of 5 visits to the laboratory, which was always kept at $24^{\circ} \mathrm{C}$, with a relative humidity of approximately $50 \%$. Visits 1 and 2 consisted of familiarizations to the maximum dynamic strength and strength endurance tests. The maximum dynamic strength value found at visit 2 was employed to prescribe the strength endurance test in the three experimental sessions. In a counterbalanced crossover fashion, participants were submitted to the following experimental conditions: control - no stretching (CON), shortduration stretching (SDS) and long-duration stretching (LDS). Both the short- and long-duration stretching protocols were applied to the quadriceps muscle group unilaterally to the dominant leg. The protocols were performed immediately after a 5-minute general warm-up in a treadmill and immediately before a specific warmup for the strength endurance test (see Figure 1). During the CON condition, participants rested quietly for a similar duration to the one that SDS lasted, and then the specific warm-up for the strength endurance test was started. The experimental sessions were separated by washout periods of 72 hours and were randomly assigned to participants through an adapted William's Latin Square model (Kuehl, 2000). All experimental sessions were individually standardized. Participants were requested to abstain from alcohol, as well as from unaccustomed and/or lower limb exercise in the 48 
hours prior to the experimental sessions. Caffeine was also avoided in the 24 hours preceding the tests. Participants arrived at the laboratory at least 2 hours following their last meal and immediately began their warm-up. Ad libitum water consumption was allowed during the sessions. The participants' maximum dynamic strength and strength endurance were evaluated during the unilateral knee extension exercise. Strength endurance was assessed through the number of repetitions performed in a protocol involving 4 sets until failure at $70 \%$ of the one-repetition maximum (1-RM).

\section{Unilateral maximum dynamic strength test}

Unilateral maximum dynamic strength (1-RM) was determined as the maximum possible weight lifted in a single and complete repetition in a specific equipment (Gervasport, Pleven, Bulgaria) using the dominant leg. Knee joint amplitude was set at $90^{\circ}$ using a goniometer. The procedures followed the American Society of Exercise Physiologists guidelines (Brown \& Weir, 2001). The participants ran for 5 minutes in a treadmill at $9 \mathrm{~km} / \mathrm{h}$ for a general warm-up. Before the tests, the participants performed a specific warm-up set of eight repetitions at approximately $50 \%$ of the estimated 1-RM followed by another set of three repetitions at $70 \%$ of the estimated 1-RM. Warm-up sets were separated by a 2-minute interval. After the completion of the second set, the participants rested for 3 minutes. Subsequent lifts were single repetitions of progressively heavier loads using the dominant leg, until failure. Unilateral maximum dynamic strength was determined as the maximum weight that could be lifted once with proper technique. The rest interval between 1-RM attempts was set at a 3-minute period, and a maximum of five
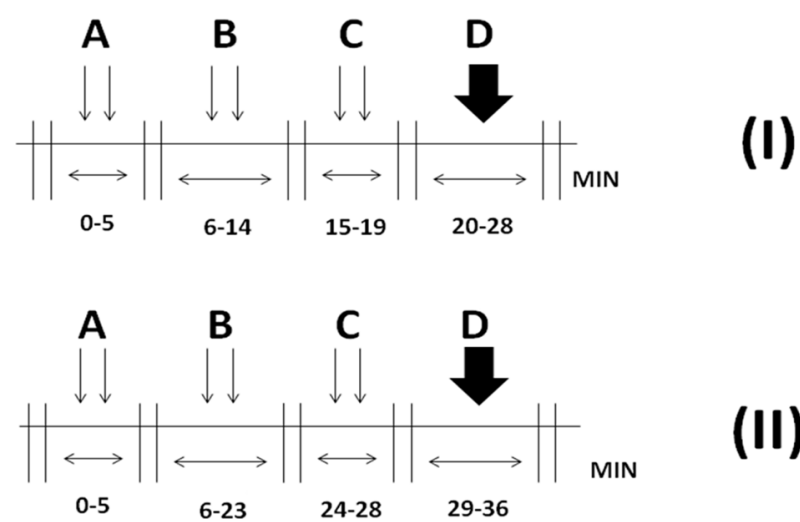

(II)

Figure 1. Overview of the testing procedures during the short-duration (I) and long-duration stretching protocols (II). A = general warm-up; $\mathrm{B}=$ stretching session; $\mathrm{C}=$ specific warm-up; $\mathrm{D}=$ unilateral knee-extension strength endurance test. attempts was allowed. All the tests were accompanied by two-experienced researchers. Increments in weight were determined according to researchers' perception. Strong verbal encouragement was provided during all lifts. The coefficient of variation across the familiarization sessions was $2.74 \%$.

\section{Unilateral strength endurance test}

Using the dominant leg, unilateral strength endurance assessment consisted of the completion of 4 sets until failure at $70 \%$ of the $1-\mathrm{RM}$ obtained in the last familiarization session. Initially, each participant performed an eight-repetition warm-up set using 50\% of the load to be used in the test. After a 2-minute rest, they performed three repetitions at $70 \%$ of the load to be used in the test. During the test, all of the sets were performed until failure, and the total number of repetitions was obtained, as well as the number of repetitions per set. For safety purposes, a 90-second rest interval was allowed between sets and the researchers were only allowed to intervene in the execution of the repetition if there was a failure. Strong verbal encouragement was provided during all repetitions. The coefficient of variation across the familiarization sessions was $7.39 \%$.

\section{Acute stretching sessions}

During the experimental sessions, participants performed 3 static stretching exercises, all of them targeting the quadriceps muscle group in the dominant leg. During the SDS, 3 sets of 30 seconds were performed for each exercise, with a 30-second interval between the sets and the exercises. During the LDS, 6 sets of $30 \mathrm{sec}-$ onds were performed for each exercise, with a 30 -second interval between the sets and the exercises. Hence, SDS lasted 9 minutes, while LDS lasted 18 minutes. During the stretching exercises, the participants were assisted by an experienced researcher and each set was performed with the amplitude being held to the point of reported discomfort. The employed stretching exercises included the kneeling quadriceps stretch, the side quadriceps stretch, as well as the standing quadriceps stretch, and are more accurately detailed elsewhere (Jeffreys, 2016).

\section{Statistical analysis}

Data were processed using basic descriptive statistics. Data normality was tested by the Shapiro-Wilk test and visually inspected (box-plots) to observe the presence of outliers. The total number of repetitions was analyzed by applying mixed models with repeated measures, assuming Condition as a fixed factor, and Participant as a random factor. The number of repetitions per set was analyzed employing mixed models with repeated measures, assuming Condition and Set as fixed factors, 
and Participant as a random factor. Tukey tests were used for post-hoc analyses. Effect sizes for the total number of repetitions were calculated using Cohen's $d$, and a $95 \%$ confidence interval $(95 \% \mathrm{CI})$ was also included. Additionally, a magnitude-based inference was conducted following previous recommendations (Batterham \& Hopkins, 2006) to detect small effects of practical importance in an applied setting. This establishes the likelihood (in percentage terms) of each experimental manipulation having a positive, trivial, or negative effect. Qualitative descriptors were assigned to the positive percentile scores as follows: < $1 \%$, almost certainly not; $1-5 \%$, very unlikely; 5-25\%, unlikely; $25-75 \%$, possibly; 75-95\%, likely; 95-99\%, very likely; > 99\%, almost certainly (Batterham \& Hopkins, 2006). Data analysis was conducted in SAS software (Version 9.3; SAS Institute, Cary, NC, USA). The level of significance was previously established at $p<.05$.

\section{Results}

Mixed model analysis did not show a main effect of Condition $(F=0.14, p=.86)$ for the total number of repetitions. Similarly, multiple comparison analysis did not reveal any differences in the total number of repetitions between CON vs. SDS ( $p=.87, d=-0.19,95 \%$ CI $[-0.94,0.55]), \mathrm{CON}$ vs. $\operatorname{LDS}(p=.99, d=-0.03$, $95 \%$ CI $[-0.77,0.71])$ or SDS vs. LDS $(p=.91$, $d=0.14,95 \%$ CI $[-0.60,0.89])$. Compared to CON, magnitude-based inference analysis revealed that changes in the total number of repetitions after SDS and LDS were mainly trivial (see Table 1).

Mixed model analysis did not show a Condition vs. Set interaction effect $(F=0.26, p=.95)$ for the number of repetitions performed in each set. However, a significant main effect of Set was observed $(F=44.74$, $p<.001)$, indicating a reduction in the number of repetitions performed across the sets. Nevertheless, the multiple comparison analysis did not show any significant differences in the number of repetitions performed in each set between the experimental conditions (all comparisons, $p>.05$; see Figure 2). Compared to $\mathrm{CON}$, magnitude-based inference analysis revealed that the changes in the number of repetitions performed in Set 1 and Set 2 after SDS and LDS were mainly trivial (Table 1). On the other hand, compared to CON, the changes in the number of repetitions performed in Set 4 after SDS and LDS were mainly negative (Table 1).

\section{Discussion}

There has been a long-stand debate about possible interference of acute stretching on subsequent strength performance. In the present study, however, we showed that an acute session of static stretching, whose duration was within the international recommendation, did not negatively affect the strength endurance of recreationally strength-trained individuals during a

Table 1

Magnitude-based inference analysis of the total number of repetitions and repetitions per set

\begin{tabular}{|c|c|c|c|c|}
\hline & Change (\%) & $\begin{array}{l}\text { Chances of treatment } \\
\text { being positive }(\%)\end{array}$ & $\begin{array}{l}\text { Chances of treatment } \\
\text { being trivial }(\%)\end{array}$ & $\begin{array}{c}\text { Chances of treatment } \\
\text { being negative }(\%)\end{array}$ \\
\hline \multicolumn{5}{|l|}{ Total repetitions } \\
\hline CON vs. SDS & -2.50 & 2 & 48 & 50 \\
\hline CON vs. LDS & -0.94 & 11 & 69 & 20 \\
\hline \multicolumn{5}{|l|}{ Repetitions 1st set } \\
\hline CON vs. SDS & +0.84 & 12 & 65 & 23 \\
\hline CON vs. LDS & -0.15 & 11 & 72 & 17 \\
\hline \multicolumn{5}{|c|}{ Repetitions 2nd set } \\
\hline CON vs. SDS & +0.83 & 31 & 51 & 18 \\
\hline CON vs. LDS & +0.47 & 43 & 48 & 9 \\
\hline \multicolumn{5}{|l|}{ Repetitions 3rd set } \\
\hline CON vs. SDS & -3.80 & 5 & 28 & 67 \\
\hline CON vs. LDS & +0.002 & 15 & 61 & 23 \\
\hline \multicolumn{5}{|c|}{ Repetitions 4th set } \\
\hline CON vs. SDS & -10.67 & 1 & 21 & 77 \\
\hline CON vs. LDS & -4.66 & 6 & 41 & 53 \\
\hline
\end{tabular}

Note. $\quad \mathrm{CON}=$ control; $\mathrm{SDS}=$ short-duration stretching; $\mathrm{LDS}=$ long-duration stretching. 


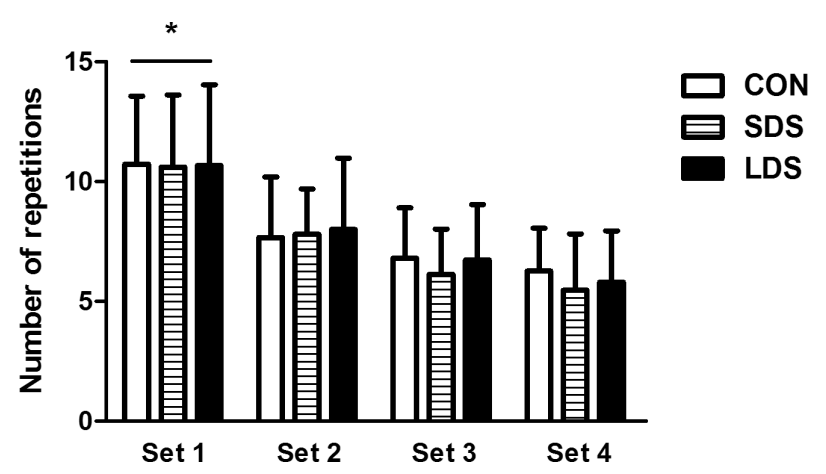

Figure 2. Number of repetitions performed in each set during the no stretching (CON), short-duration stretching (SDS) and long-duration stretching (LDS) conditions in recreationally trained individuals. The asterisk indicates a significant difference $(p<.05)$ compared to Set 2, Set 3 and Set 4.

unilateral knee extension exercise. Importantly, we also showed that doubling the duration of this session did not cause a further impairment on strength endurance.

The detrimental effects of acute stretching have been consistently shown in some forms of muscle strength manifestation, such as jumping power (Pinto, Wilhelm, Tricoli, Pinto, \& Blazevich, 2014), peak torque (Babault, Bazine, Deley, Paizis, \& Lattier, 2015), sprinting speed (Paradisis et al., 2014) and 1-RM (Gergley, 2013). In fact, meta-analysis data support the ergolytic effects of stretching, with mean decrements of 10 to $30 \%$ in strength performance (Shrier, 2004). It is speculated that both mechanical and neural factors might be contributing to the loss of force induced by stretching. In this direction, previous studies have shown that impaired isometric torque production of the hamstrings was most prominent at shorter muscle lengths, which suggests that stretching may affect the length-tension relationship of skeletal muscle, and then its contractile properties (Herda, Cramer, Ryan, McHugh, \& Stout, 2008). Using surface electromyography, other authors have demonstrated decreased muscle activation induced by stretching, suggesting a possible inhibitory interference from the central nervous system (Cramer et al., 2005). The exact mechanisms underpinning both neural and mechanical factors still merit further clarification.

On the other hand, the number of studies investigating the effects of stretching on strength endurance is reduced, and their results are controversial. Initial studies on this matter showed that 15 minutes of passive static stretching promoted a negative and consistent effect on strength endurance during a leg curl exercise (Nelson, Kokkonen, \& Arnall, 2005). Subsequent well-controlled studies have also shown that different stretching protocols (static stretching, ballistic stretching and proprioceptive neuromuscular facilitation) impaired strength endurance, in a similar magnitude, when compared to a control condition (Barroso et al., 2012). Others, however, did not observe decrements in performance with a static stretching protocol consisting of 3 sets of 20 seconds, although a proprioceptive neuromuscular facilitation protocol negatively affected bench press strength endurance (Franco, Signorelli, Trajano, \& de Oliveira, 2008). Similarly, a proprioceptive neuromuscular facilitation protocol, but not static stretching, was shown to impair bench press and knee extension strength endurance in all the different intensities tested (40, 60 and 80\% 1-RM; Gomes et al., 2011). Conversely, other studies did not demonstrate significant differences in strength endurance when a leg curl exercise was preceded by a proprioceptive neuromuscular facilitation protocol (lasting $\sim 150$ seconds) compared to a control condition (Keese et al., 2013). More recent studies also did not show any negative influence from static stretching protocols lasting 150 and 180 seconds on the bench press (Ribeiro et al., 2014) and squat strength endurance (Heisey \& Kingsley, 2016), respectively. It is difficult to explain the divergence of results, but one might speculate that the heterogeneity inherent to the experimental designs, the varied level of training of the participants, as well as the differences in the stretching protocols and exercise tests employed are contributing confounding factors.

Considering these discrepant results, the present investigation examined the effects of an acute static stretching session on strength endurance. Moreover, if stretching is an interfering factor on strength performance, one should expect a greater interference from longer stretching durations on strength performance. However, in the current study, no significant differences occurred in the total number of repetitions after SDS or LDS when compared to CON. The relative changes as well as the magnitude-based inference results (see Table 1) further support the notion that neither the SDS nor the LDS protocols had any influence on the total number of repetitions compared to $\mathrm{CON}$, and that changes promoted by these protocols were mainly trivial. Therefore, we are confident to affirm that an acute session of static stretching had no effect on strength endurance, independent of its duration. These results are in contrast with those demonstrating an interference effect of stretching on strength performance (Barroso et al., 2012; Franco et al., 2008; Gomes et al., 2011; Nelson, Kokkonen, \& Arnall, 2005). One plausible explanation to these findings relies on the time taken between the end of the stretching protocols and the start of the strength endurance test. In this sense, Ryan et al. (2008) observed 
that 4 and 8 minutes of plantar flexion passive stretching induced an acute and significant decrement in the rate of torque development during a maximal voluntary contraction performed immediately post-stretching. However, when the rate of torque development was evaluated 10, 20 and 30 minutes post-stretching, no changes were shown. In the present study, after both the execution of the stretching protocols, a specific warm-up was performed. At total, approximately 5 minutes separated the stretching session from the strength test, and hence, such a strategy may help to explain the absence of an acute interference observed in our study. On the other hand, it is equally important to remind that the specific warm-up is an effective approach to prevent exercise-related injuries (Herman, Barton, Malliaras, \& Morrissey, 2012), besides being a pre-exercise strategy with international recommendations (Garber et al., 2011). In fact, despite no tool (i.e., goniometer) was employed to evaluate the change in range of motion induced by stretching, the stretching procedure was the same as that recommended by the American College of Sports Medicine (i.e., each set was performed with the amplitude being held to the point of reported discomfort). Taken together these findings suggest that the acute interference phenomena may only occur if the strength requirement is immediately preceded by stretching. Accordingly, if proper and safety practices are performed, such as the specific warm-up, the stretching-induced strength endurance decrements may not occur. Future studies need to test such a hypothesis.

This study is not without limitations. Firstly, the employed exercise test does not necessarily reflect a real-world strength training session, where multiple exercises and/or more sets are typically performed. In this sense, although our magnitude based-inference analysis did show that changes in the total number of repetitions with SDS and LDS were mainly trivial, when this analysis was applied to each set, both the SDS and LDS protocols were shown to promote mainly negative changes in the number of repetitions performed in the last set of exercise. Thus, it is still possible that performance during high-volume strength training sessions may be acutely affected by stretching. More studies are necessary to examine this hypothesis and to employ strength testing protocols with a broad range of duration, intensity, repetitions and resting times. Secondly, only the static stretching approach was tested during the present study. The real effect of different stretching methods (e.g., ballistic, proprioceptive neuromuscular facilitation) on strength endurance, as well as the influence of different durations of such methods on performance, constitute a relevant research question to be further explored.

\section{Conclusions}

To conclude, regardless of its duration, an acute session of static stretching did not influence the strength endurance of recreationally strength-trained individuals during a unilateral knee extension exercise. These results suggest that static stretching protocols within the international recommendations may be safely employed before a strength training session without the risk of performance impairment, and therefore, possibly without the risk of interference on some exercise adaptations, such as muscle hypertrophy. For purposes of performance optimization and injury prevention, individuals engaging in an exercise training session should follow the international guidelines for stretching and pre-exercise warm-up.

\section{Conflict of interest}

There were no conflicts of interest.

\section{References}

Alizadeh Ebadi, L., \& Çetin, E. (2018). Duration dependent effect of static stretching on quadriceps and hamstring muscle force. Sports, 6, 24.

Babault, N., Bazine, W., Deley, G., Paizis, C., \& Lattier, G. (2015). Direct relation of acute effects of static stretching on isokinetic torque production with initial flexibility level. International Journal of Sports Physiology and Performance, 10, 117-119.

Barroso, R., Tricoli, V., Santos Gil, S. D., Ugrinowitsch, C., \& Roschel, H. (2012). Maximal strength, number of repetitions, and total volume are differently affected by static-, ballistic-, and proprioceptive neuromuscular facilitation stretching. Journal of Strength and Conditioning Research, 26, 2432-2437.

Batterham, A. M., \& Hopkins, W. G. (2006). Making meaningful inferences about magnitudes. International Journal of Sports Physiology and Performance, 1, 50-57.

Brown, L. E., \& Weir, J. P. (2001). ASEP procedures recommendation I: Accurate assessment of muscular strength and power. Journal of Exercise Physiology, 4(3), 1-21.

Cramer, J. T., Housh, T. J., Weir, J. P., Johnson, G. O., Coburn, J. W., \& Beck, T. W. (2005). The acute effects of static stretching on peak torque, mean power output, electromyography, and mechanomyography. European Journal of Applied Physiology, 93, 530-539.

Franco, B. L., Signorelli, G. R., Trajano, G. S., \& de Oliveira, C. G. (2008). Acute effects of different stretching exercises on muscular endurance. Journal of Strength and Conditioning Research, 22, 1832-1837.

Garber, C. E., Blissmer, B., Deschenes, M. R., Franklin, B. A., Lamonte, M. J., Lee, I.-M., ... Swain, D. (2011). Quantity and quality of exercise for developing and maintaining cardiorespiratory, musculoskeletal, and neuromotor 
fitness in apparently healthy adults. Medicine \& Science in Sports \& Exercise, 43, 1334-1359.

Gergley, J. C. (2013). Acute effect of passive static stretching on lower-body strength in moderately trained men. Journal of Strength and Conditioning Research, 27, 973-977.

Gomes, T. M., Simão, R., Marques, M. C., Costa, P. B., \& da Silva Novaes, J. (2011). Acute effects of two different stretching methods on local muscular endurance performance. Journal of Strength and Conditioning Research, 25, 745-752.

Heisey, C. F., \& Kingsley, J. D. (2016). Effects of static stretching on squat performance in Division I female athletes. International Journal of Exercise Science, 9, 359-367.

Herda, T. J., Cramer, J. T., Ryan, E. D., McHugh, M. P., \& Stout, J. R. (2008). Acute effects of static versus dynamic stretching on isometric peak torque, electromyography, and mechanomyography of the biceps femoris muscle. Journal of Strength and Conditioning Research, 22, 809-817.

Herman, K., Barton, C., Malliaras, P., \& Morrissey, D. (2012). The effectiveness of neuromuscular warm-up strategies, that require no additional equipment, for preventing lower limb injuries during sports participation: A systematic review. BMC Medicine, 10, 75.

Islamoglu, I., Atan, T., Unver, S., \& Cavusoglu, G. (2016). Effects of different durations of static stretching on flexibility, jumping, speed and agility performance. Anthropologist, 23, 454-461.

Jeffreys, I. (2016). Warm-up and flexibility training. In G. G. Haff \& N. T. Triplett (Eds.), Essentials of strength training and conditioning (4th ed., pp. 317-350). Champaign, IL: Human Kinetics.

Julious, S. A. (2005). Sample size of 12 per group rule of thumb for a pilot study. Pharmaceutical Statistics, 4, 287-291.

Junior, R. M., Berton, R., De Souza, T. M., Chacon-Mikahil, M. P., \& Cavaglieri, C. R. (2017). Effect of the flexibility training performed immediately before resistance training on muscle hypertrophy, maximum strength and flexibility. European Journal of Applied Physiology, 117, 767-774.

Keese, F., Farinatti, P., Massaferri, R., Matos-Santos, L., Silva, N., \& Monteiro, W. (2013). Acute effect of proprioceptive neuromuscular facilitation stretching on the number of repetitions performed during a multiple set resistance exercise protocol. Journal of Strength and Conditioning Research, 27, 3028-3032.

Kuehl, R. O. (2000). Repeated measures design. In R. O. Kuehl (Ed.), Design of experiments: Statistical principles of research design and analysis (2nd ed., pp. 576-586). London, United Kingdom: Duxbury-Thomson Learning.
Marek, S. M., Cramer, J. T., Fincher, A. L., Massey, L. L., Dangelmaier, S. M., Purkayastha, S., ... Culbertson, J. Y. (2005). Acute effects of static and proprioceptive neuromuscular facilitation stretching on muscle strength and power output. Journal of Athletic Training, 40, 94-103.

Nelson, A. G., Kokkonen, J., \& Arnall, D. A. (2005). Acute muscle stretching inhibits muscle strength endurance performance. Journal of Strength and Conditioning Research, 19, 338-343.

Paradisis, G. P., Pappas, P. T., Theodorou, A. S., Zacharogiannis, E. G., Skordilis, E. K., \& Smirniotou, A. S. (2014). Effects of static and dynamic stretching on sprint and jump performance in boys and girls. Journal of Strength and Conditioning Research, 28, 154-160.

Pinto, M. D., Wilhelm, E. N., Tricoli, V., Pinto, R. S., \& Blazevich, A. J. (2014). Differential effects of 30- vs. 60 -second static muscle stretching on vertical jump performance. Journal of Strength and Conditioning Research, 28, 3440-3446.

Ribeiro, A. S., Romanzini, M., Dias, D. F., Ohara, D., da Silva, D. R., Achour, A., Jr, ... Cyrino, E. S. (2014). Static stretching and performance in multiple sets in the bench press exercise. Journal of Strength and Conditioning Research, 28, 1158-1163.

Robbins, D. W., Marshall, P. W., \& McEwen, M. (2012). The effect of training volume on lower-body strength. Journal of Strength and Conditioning Research, 26, 34-39.

Rossi, L. P., Pereira, R., Simão, R., Brandalize, M., \& Gomes, A. R. (2010). Influence of static stretching duration on quadriceps force development and eletromyographic activity. Human Movement, 11, 137-143.

Ryan, E. D., Beck, T. W., Herda, T. J., Hull, H. R., Hartman, M. J., Stout, J. R., \& Cramer, J. T. (2008). Do practical durations of stretching alter muscle strength? A doseresponse study. Medicine \& Science in Sports \& Exercise, 40, 1529-1537.

Shrier, I. (2004). Does stretching improve performance? A systematic and critical review of the literature. Clinical Journal of Sport Medicine, 14, 267-273.

Simic, L., Sarabon, N., \& Markovic, G. (2013). Does preexercise static stretching inhibit maximal muscular performance? A meta-analytical review. Scandinavian Journal of Medicine and Science in Sports, 23, 131-148.

Sooneste, H., Tanimoto, M., Kakigi, R., Saga, N., \& Katamoto, S. (2013). Effects of training volume on strength and hypertrophy in young men. Journal of Strength and Conditioning Research, 27, 8-13. 